\title{
MEDICAL GAS
}

RESEARCH

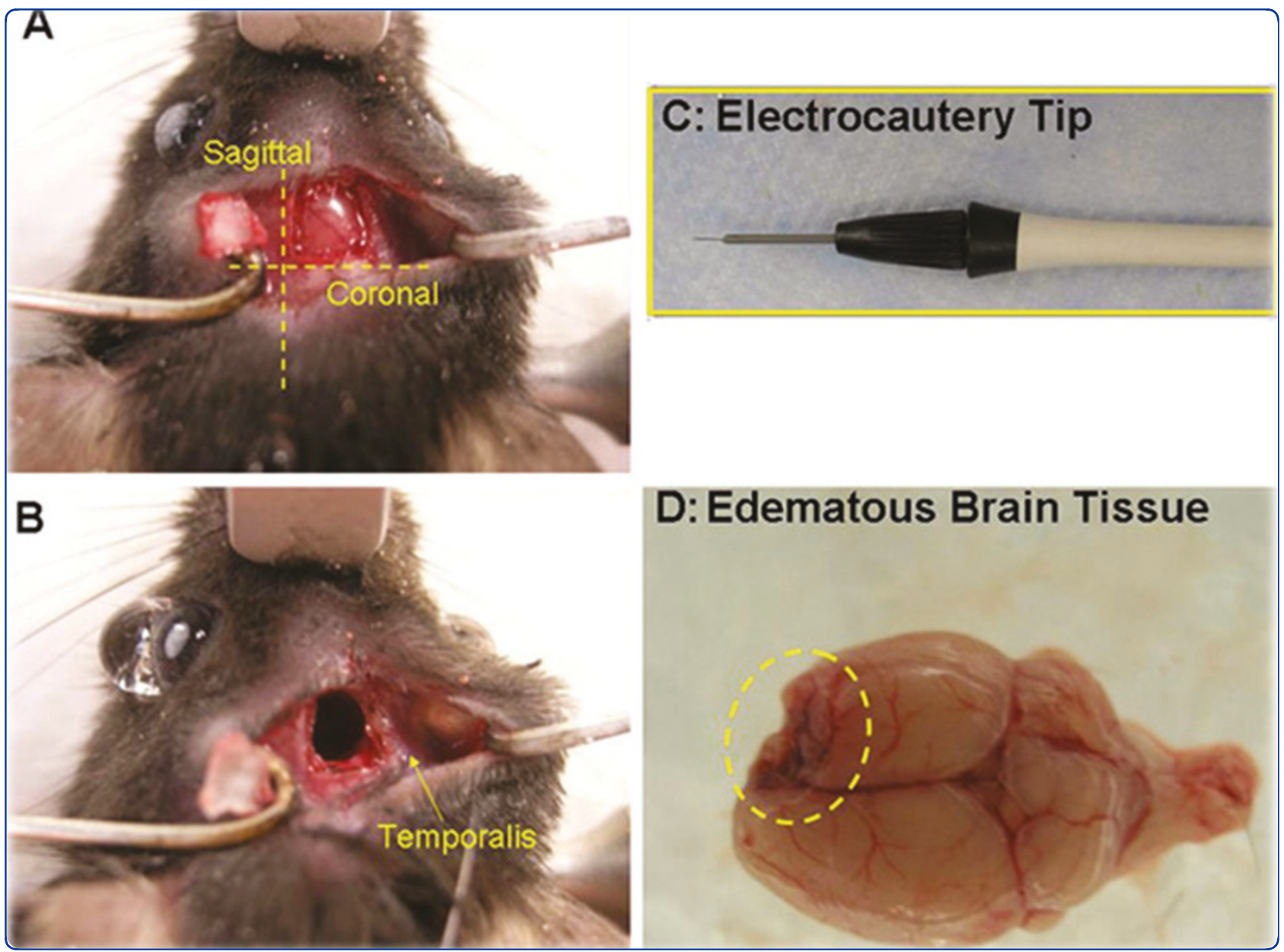

\section{Hydrogen is neuroprotective against surgically induced brain injury}

Eckermann et al. 


\title{
Hydrogen is neuroprotective against surgically induced brain injury
}

\author{
Jan M Eckermann ${ }^{1}$, Wanqiu Chen ${ }^{2}$, Vikram Jadhav², Frank PK Hsu ${ }^{1}$, Austin RT Colohan ${ }^{1}$, Jiping Tang ${ }^{2}$ and \\ John H Zhang ${ }^{1,2,3^{*}}$
}

\begin{abstract}
Background: Neurosurgical operations cause unavoidable damage to healthy brain tissues. Direct surgical injury as well as surgically induced oxidative stress contributes to the subsequent formation of brain edema. Therefore, we tested the neuroprotective effects of hydrogen $\left(\mathrm{H}_{2}\right)$ in an established surgical brain injury (SBI) model in rats.

Materials and methods: Adult male Sprague - Dawley rats (weight 300-350g) were divided into three groups to serve as sham operated, SBI without treatment, and SBI treated with $\mathrm{H}_{2}(2.9 \%)$. Brain water content,

myeloperoxidase (MPO) assay, lipid peroxidation (LPO), and neurological function were measured at $24 \mathrm{hrs}$ after SBI.

Results: SBI resulted in localized brain edema ( $p=<0.001$ ). Hydrogen (2.9\%) administered concurrently with surgery significantly decreased the formation of cerebral edema $(p=0.028)$ and improved neurobehavioral score $(p=0.022)$. However, hydrogen treatment failed to reduce oxidative stress (LPO assay) or inflammation (MPO assay) in brain tissues.
\end{abstract}

Conclusions: Hydrogen appears to be promising as an effective, yet inexpensive way to reduce cerebral edema caused by surgical procedures. Hydrogen has the potential to improve clinical outcome, decrease hospital stay, and reduce overall cost to patients and the health care system.

\section{Introduction}

In 2008, close to 800,000 patients underwent neurosurgical procedures in the United States. This figure encompasses emergent, life-preserving surgeries, as well as planned procedures. Neurosurgical operations have the potential to cause unavoidable healthy brain tissue damage as a result through application of pressure, tissue stretching, hemorrhage, and the use of electrocautery $[1,2]$. This damage occurs despite intraoperative adjunct treatments such as administration of steroids and mannitol, irrespective of the procedure being planned or emergent. Even standard microsurgical technique has the potential to cause damage to the surrounding brain and lead to early complications such as edema and local ischemia [3]. Furthermore so-called "minimally-invasive" operations and endoscopic-assisted procedures are not immune to damage the surrounding

\footnotetext{
* Correspondence: johnzhang3910@yahoo.com

${ }^{1}$ Department of Neurosurgery, Loma Linda University Medical Center, 11234 Anderson Street, Loma Linda, CA 92354, USA

Full list of author information is available at the end of the article
}

structures [4]. Moreover, radiosurgery, which has the least invasive method of addressing intracranial lesions, has the potential to cause severe cerebral edema [5].

Some clinical-used therapeutic agents have shown to be neuroprotective in experimental models [6]. Although pretreatment of the subjects may be an option for planned procedures, it proves to be not useful in the emergent setting for obvious reasons. The ideal therapy would be to start at the time when the neurosurgical intervention is carried out, and is completed at the time when the intervention is completed. Furthermore, this therapy should have a low profile with regard to toxicity, adverse effects, drug-drug interaction, and should also be cost-effective.

Recent data suggests that hydrogen $\left(\mathrm{H}_{2}\right)$ has neuroprotective effects in an experimental model of cerebral ischemia [7]. Inhaled hydrogen was shown to decrease the infarct size by reducing reactive oxygen species formation. We hypothesize that hydrogen, inhaled concomitantly with the neurosurgical intervention, has potential to reduce brain edema and improve

\section{Biomed Central}


neurological function in an established surgical brain injury model [8].

\section{Materials and methods}

This protocol was evaluated and approved by the Institutional Animal care and Use Committee at Loma Linda University, Loma Linda, CA.

\section{The SBI model}

The SBI model in rodents has been described previously [8]. Briefly, the rationale is that a controlled insult - a partial right frontal lobectomy - mimics standard surgical procedures. By using fixed coordinates (see below), the repeatability of this procedure is insured. By creating a lesion close to the right primary sensory and motor cortices, it is possible to observe impairments in neurological function. Both sensory as well as motor systems are affected and are quantifiable with established, objective tests. Brain water content measurement after sacrificing the animal assures an objective, quantitative method to evaluate edema formation.

\section{Surgical Procedure}

Adult male Sprague - Dawley rats (weight 300-350 g) were used for this study. The rodent model of SBI was used as described previously [8]. All animals were intubated and subject to general anesthesia, using 3\% halothane for induction and $1 \%$ for maintenance. A square cranial window was drilled such that the left lower corner of the square was at the bregma. The dura was opened and reflected to expose the underlying right frontal lobe. Using a sharp blade, two incisions were made along the saggital and coronal planes leading away from the bregma to limit the partial lobectomy to $2 \mathrm{~mm}$ lateral to the saggital plane and $1 \mathrm{~mm}$ rostral to the coronal plane. The depths of the resection extended to the base of the anterior fossa. Hemostasis was achieved by using saline irrigation, packing, and electrocautery. Sham surgery included only craniotomy and replacement of the bone flap without dural penetration. Animals were sacrificed at 24 hours. The 24 hour time point appears to be the time of peak edema formation in rodents, making it thus the prime target for any therapy aimed at edema reduction.

All animals were intubated for sixty minutes regardless of the length of the operation and group. This allows for a constant in the length of intubation and hydrogen administration. No surgery exceeded sixty minutes.

\section{Treatment Groups}

$\mathrm{H}_{2}$ was administered via the endotrachial tube. A tank premixed with $2.9 \%$ hydrogen, $21 \%$ oxygen and nitrogen balance was used for the treatment group and sham
(2.9\%, Praxair, CA, USA). The control group received $21 \%$ oxygen and nitrogen balance. Treatment time consisted of 60 minutes for all groups. The tubing was checked for leaks and adequate concentrations of hydrogen at the endotrachial tube prior to the procedure using a Hydrogen-meter $\left(\mathrm{H}_{2}\right.$ Scan, CA, USA).

\section{Brain Water Content}

The animals were sacrificed under deep anesthesia at 24 hours after frontal lobe injury. The brains were removed and divided into frontal ipsilateral, frontal contralateral, parietal ipsilateral, parietal contralateral, brain stem and cerebellum on ice. These parts were weighed immediately (wet weight) and weighed again after drying in an oven at $105^{\circ} \mathrm{C}$ for 48 hours (dry weight) as described previously [8]. The percent of water content was calculated as [(wet weight - dry weight)/wet weight] $\times 100 \%$. The number of animals used in each group for brain edema study was control $(\mathrm{n}=12)$, sham $(\mathrm{n}=8)$, hydrogen-treated $(\mathrm{n}=12)$.

\section{Myeloperoxidase Assay}

Methodology for the myeloperoxidase (MPO) assay has been described previously [9]. Briefly, frozen samples $(\mathrm{n}=18)$ were cut into 0.1-0.2 g pieces and homogenized with Tris- $\mathrm{HCl}$ and $\mathrm{Na}^{+}$buffer. The samples were then centrifuged at $14,000 \mathrm{~g}$ for 30 minutes. The supernatants were discarded and the samples were resuspended in $0.5 \% \mathrm{HTAB} / \mathrm{K}^{+}$buffer. Several cycles of freezing in liquid nitrogen followed by sonication at $25^{\circ} \mathrm{C}$ were performed, before spectrophotometrical analysis at $460 \mathrm{~nm}$. The value was divided by the exact weight of the sample and expressed in MPO activity Unit/gram.

\section{Lipid Peroxidation Assay}

The method for Lipid Peroxidase (LPO) Assay has been described in detail previously [10]. Briefly, animals ( $\mathrm{n}=$ 18) were anesthetized and brain samples were collected at 24 hours after SBI. The level of lipid peroxidation products (malondialdehyde [MDA]) in the right cerebral cortexes was measured using a LPO-586 kit (OxisResearch; Portland, OR). Right frontal cerebral cortexes were homogenized in $20 \mathrm{mmol} / \mathrm{L}$ phosphate buffer $(\mathrm{pH}$ 7.4) with $0.5 \mathrm{M}$ butylated hydroxytoluene in acetonitrile. The homogenates were centrifuged at 20,800 g for 10 min at $4^{\circ} \mathrm{C}$ and the supernatants were collected. Protein concentration was measured by DC protein assay (Bio$\mathrm{Rad})$ and the samples were reacted with a chromogenic reagent at $45^{\circ} \mathrm{C}$ for $60 \mathrm{~min}$. After incubation, the samples were centrifuged at $20,800 \mathrm{~g}$ for $10 \mathrm{~min}$ at $4^{\circ} \mathrm{C}$ and supernatants were measured at $586 \mathrm{~nm}$. The level of MDA was calculated as picomoles per milligram protein according to the standard curve. 


\section{Neurological Scoring}

We used a 21-point score adapted from the one developed for stroke by Garcia et al. [11] as presented in Table 1. Furthermore, we used the wire-hang and beam balance tests for assessment of balance, strength, and coordination. The wire-hang and beam balance test were assessed based on a three point scale with dependence on time and/or reaching the platform. $0=$ less than 15 seconds, no movement towards platform, $1=$ less than 40 seconds, no movement towards platform, 2 = less than or equal to half distance to platform within 40 seconds, and $3=$ reaching platform within 40 seconds. Three trials were performed while animals were receiving a five minute rest period in between trials. The final score was the average of three trials.

\section{Statistical Analysis}

All data were collected in SigmaPlot and analyzed with SigmaStat (both Systat Software, Inc, San Jose, CA). The one-way ANOVA test was used to calculate statistical significance between samples. P value below 0.05 was considered as statistically significant.

\section{Results}

\section{Outcome after SBI}

SBI results in localized brain edema (Figure 1A). This simply demonstrates the validity of the model. Hydrogen (2.9\%) administered concurrently with surgery significantly decreased the formation of cerebral edema (Figure 1B) evidenced by the decrease in brain water content.

Twenty-four hours after the operation, all animals underwent standardized, blinded neurological evaluation. The sensory as well as motor evaluation showed a significant improvement in the hydrogen treatment groups compared to the control (Figures 2A-C).

\section{Chemical Analysis}

Lipid peroxidation (LPO) assay did not show a decrease in oxidative stress in the hydrogen group. Paradoxically, the amount of oxidative stress increased compared to the sham and control group (Figure 3A).

Myeloperoxidase (MPO) assay was unable to demonstrate a significant attenuation of the inflammation associated with SBI. There was no statistically significant difference between treatment and control groups (Figure 3B).

\section{Discussion}

Vasogenic edema, resulting after breakdown of the blood brain barrier, is often associated with mass lesions and is encountered frequently clinically [12]. This happens not only during pathological processes, such as a tumor growth [13], but also occurs in normal healthy brain tissues caused by a neurosurgical manipulation [2]. We have observed in this study that localized brain edema occurred after SBI in rats, which leads to poor neurological function, accompanied by increase of lipid peroxidation in the injured brain tissues.

Inhaled $2.9 \%$ hydrogen during 60 minutes of surgical procedures significantly reduced brain edema and improved neurological function. This study provides evidence that inhaled hydrogen could be an easy, economic, convenient, and applicable approach for anesthesiologists and neurosurgeons to reduce surgery induced brain edema and tissue injury.

Even though we have observed positive results on the clinical outcomes, we could not confirm the mechanisms of hydrogen induced neuroprotection reported by others [7]. It has been postulated that hydrogen reacts with reactive oxygen species causing a decreases in oxidative stress at the tissue level and thus decreasing the amount of blood brain barrier breakdown [7]. The

Table 1 The Garcia Neurological Scoring Scale

\begin{tabular}{|c|c|c|c|c|c|}
\hline & Test & 3 & 2 & 1 & 0 \\
\hline$I$ & $\begin{array}{l}\text { Spontaneous } \\
\text { activity }\end{array}$ & $\begin{array}{l}3-4 \text { walls, } 1-2 \text { walls plus raise } \\
\text { on hindlimbs }\end{array}$ & 1-2 walls & Minimal movement & Akinesia \\
\hline II & Side stroking & Bilateral brisk & $\begin{array}{l}\text { Bilateral weak or ipsilateral strong and } \\
\text { contralateral weak }\end{array}$ & Unilateral & No response \\
\hline III & $\begin{array}{l}\text { Vibrissae } \\
\text { touch }\end{array}$ & Bilateral brisk & $\begin{array}{l}\text { Bilateral weak or ipsilateral strong and } \\
\text { contralateral weak }\end{array}$ & Unilateral & No response \\
\hline IV & $\begin{array}{l}\text { Limb } \\
\text { symmetry }\end{array}$ & $\begin{array}{l}\text { Forelimb and hindlimb } \\
\text { extended }\end{array}$ & Mid flexion of forelimb & $\begin{array}{l}\text { Contralateral forelimb flexed with } \\
\text { hindlimb extended }\end{array}$ & $\begin{array}{l}\text { Contralaterally } \\
\text { flexed }\end{array}$ \\
\hline $\mathrm{v}$ & $\begin{array}{l}\text { Lateral } \\
\text { turning }\end{array}$ & Bilateral turning $>450$ & Bilateral turning $<450$ & Unilateral turning & No turning \\
\hline$\overline{\mathrm{VI}}$ & $\begin{array}{l}\text { Forelimb } \\
\text { walking }\end{array}$ & Brisk forward & Moves toward on side & Moves in circle & $\begin{array}{l}\text { Cannot move } \\
\text { forelimbs }\end{array}$ \\
\hline VII & Climbing & Climbs to top & $\begin{array}{l}\text { Climbs up weak or strong but not to } \\
\text { top }\end{array}$ & Moves laterally or downward & $\begin{array}{l}\text { Weak grip and } \\
\text { falls off }\end{array}$ \\
\hline
\end{tabular}

Note: The neurological evaluation system adapted from Garcia et al. This system was applied to all groups in a blinded fashion. 


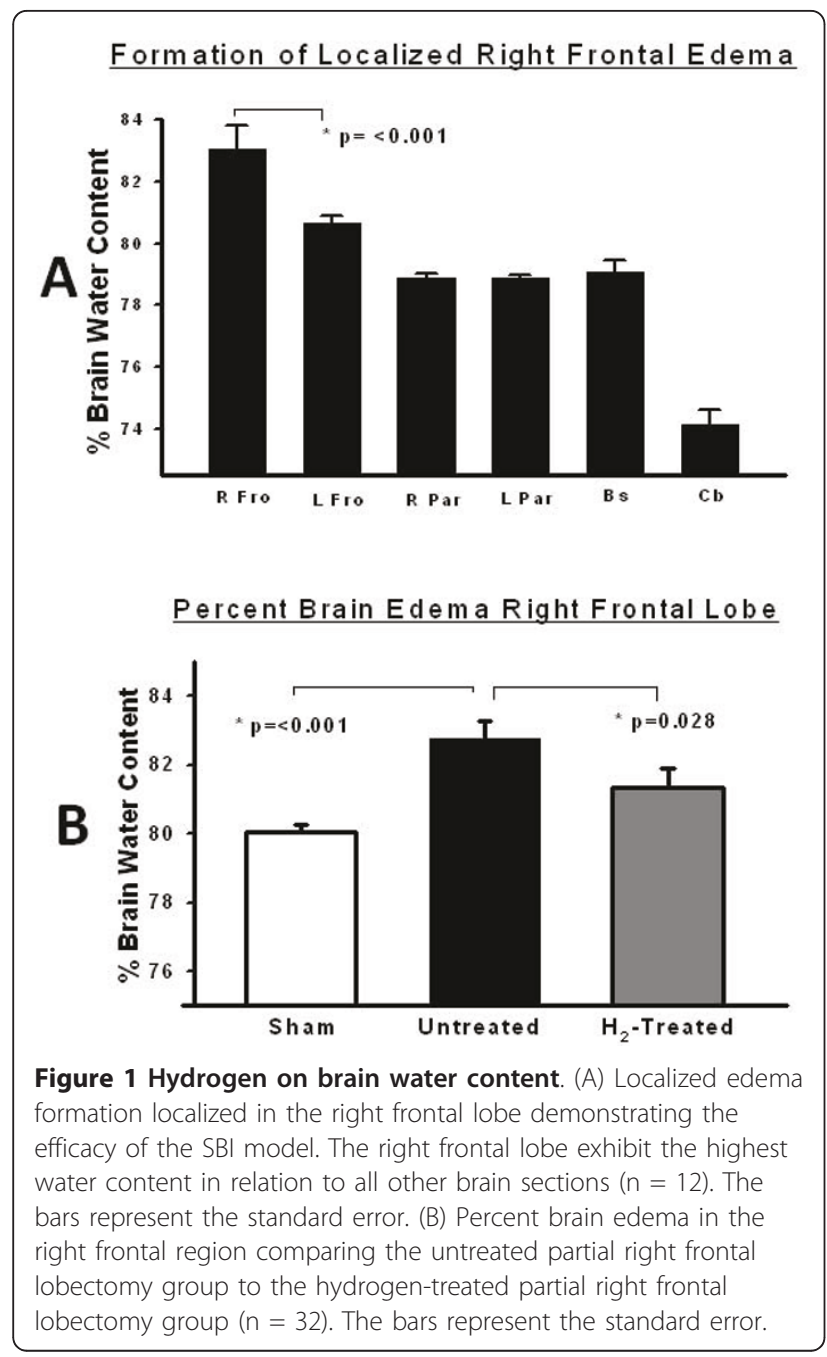

decrease in blood brain barrier breakdown in turn results in the decreases vasogenic edema formation [14]. We measured lipid peroxidation, the final outcome of oxidative stress [15]. SBI produced localized brain edema accompanied by an increase of lipid peroxidation in this rat model. Inhaled hydrogen reduced formation of brain edema, but not only did it fail to reduce, but rather it enhanced lipid peroxidation significantly. This observation is not consistent with a previous report that hydrogen is a scavenger of free radicals [7]. Somehow, our results in this study are consistent with one of our previous publications that hydrogen failed to reduce lipid peroxidation in a neonatal hypoxia-ischemia rat pup model [16]. Even though it is possible that hydrogen scavenges selectively hydroxyl radicals [7], the overall effect of hydrogen on lipid peroxidation is either ineffective [16] or potentiating, as observed in this study. It is not clear whether there is an interaction between halothane and hydrogen. However, Ohsawa et al. demonstrated $\mathrm{H}_{2}$

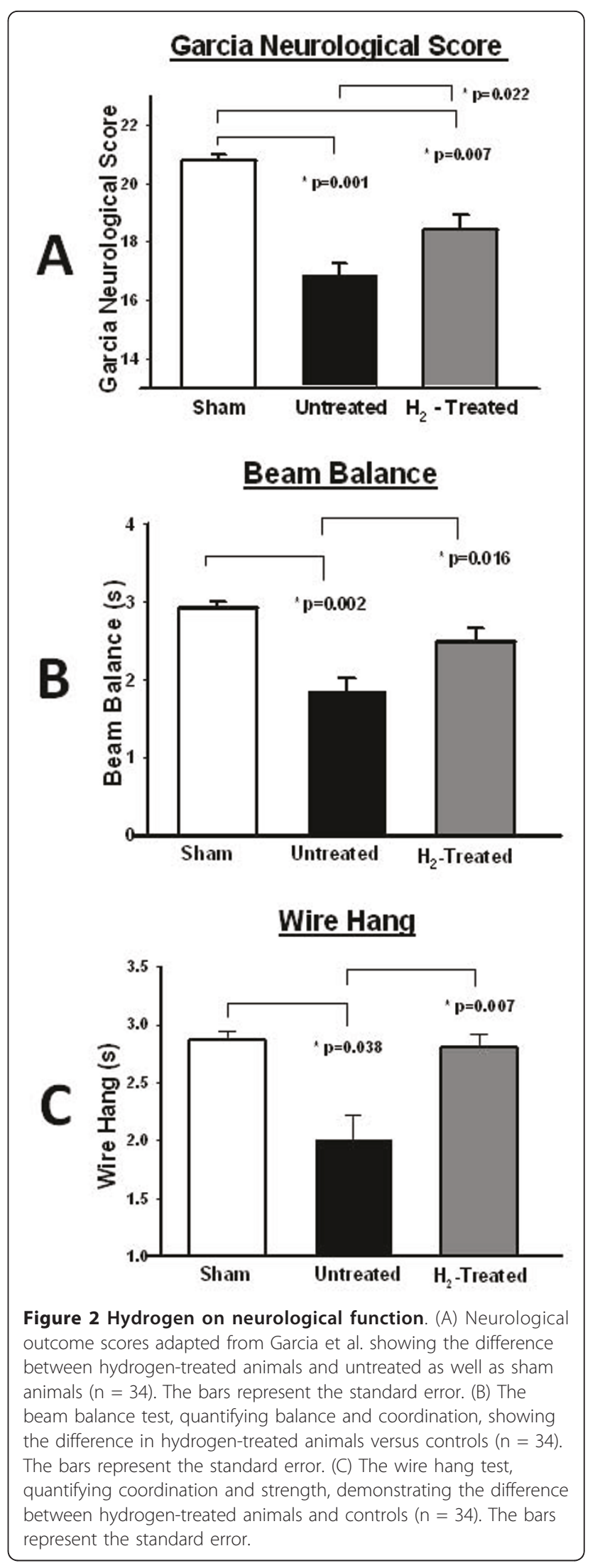




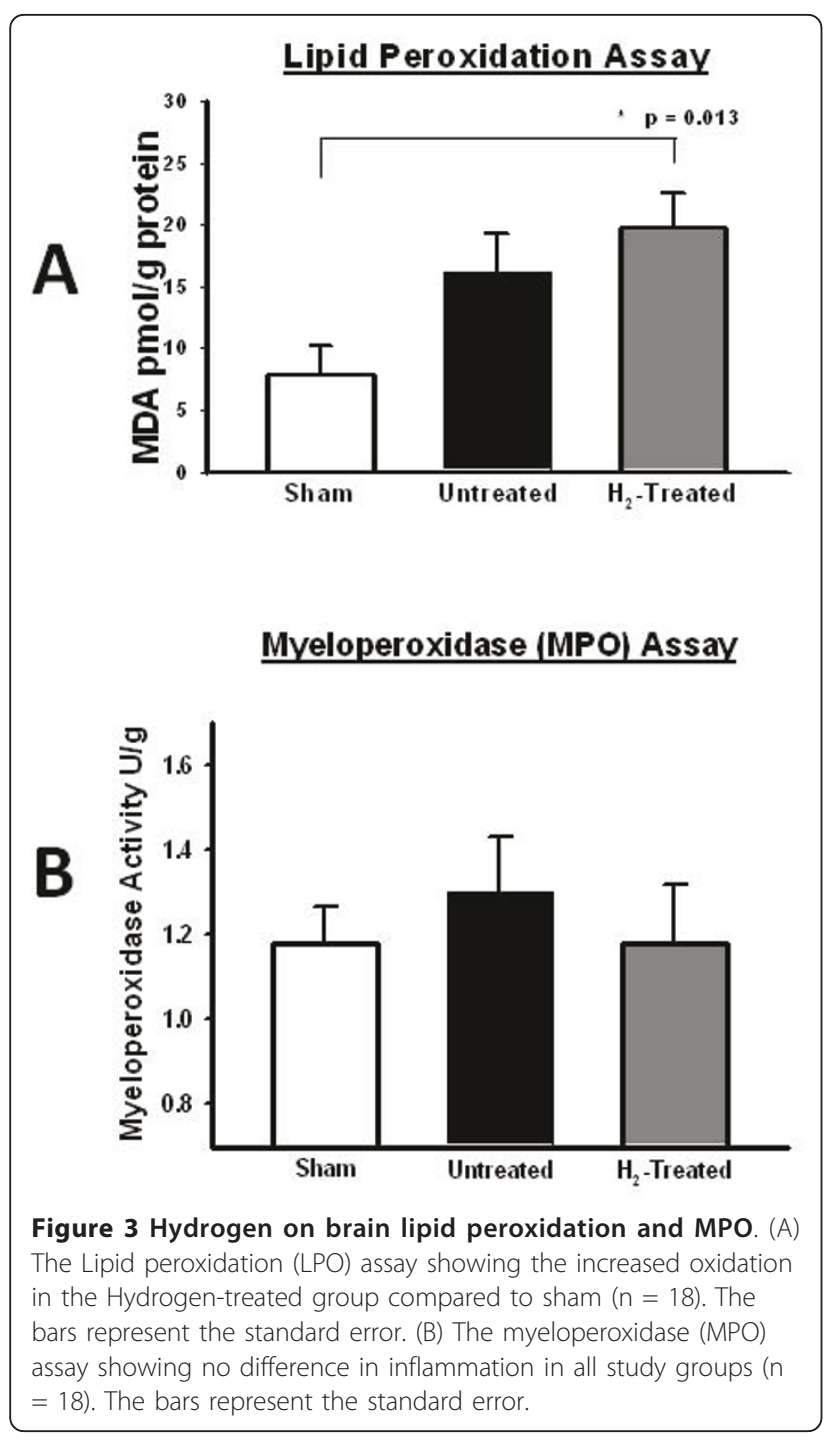

protects cultured cells against oxidative stress, without the existence of halothane [7]. It suggests that hydrogeninduced neuroprotection is halothane-independent.

Because hydrogen failed to reduce lipid peroxidation, we examined its effect on inflammation. It has been reported that inflammation contributes to secondary brain injury in stroke and traumatic brain injury [17], and anti-inflammatory strategy achieved marked brain protection in different experimental models [18]. Therefore it is highly likely that surgical brain injury is partially mediated by inflammatory responses in rats and in mice. Similar observations of surgical induced brain injury mediated by inflammation have been reported. Because neural inflammation can be detected by MPO assay [9], we measured MPO in sham, SBI, and SBI treated with hydrogen rats. We found, however, that MPO was not increased after surgical brain injury and inhaled hydrogen did not increase or decrease MPO activity.

Inhaled hydrogen gas has been proven to be safe in humans (divers) at low concentrations [19]. Even though pure hydrogen is safe, the threshold for combustion of hydrogen if mixed with oxygen lies at four percent [20]. It has been shown that with $2-4 \%$ of $\mathrm{H}_{2}$ inhalation significantly reduced infarct volume against middle cerebral artery occlusion [7]. In our study with $2.9 \%$ and for only one hour, we are clearly observing a phenomenon of decreasing cerebral edema formation as well as improved functional outcome in animals treated with inhaled hydrogen. Therefore, hydrogen appears to be promising as an effective, yet inexpensive way to reduce cerebral edema. The non-toxicity of low-dose hydrogen [3] coupled with its availability and ease of administration makes a clinical translation very simple and foreseeable in the near future. Based on the results observed in this study, perioperative use of hydrogen has the potential to improve clinical outcome, decrease overall healthcare cost and length of hospital stay, as well as increased patient satisfaction. Further investigations are necessary to elucidate the exact mechanism in which hydrogen exerts its protection on neuronal tissues. Our study only assessed the effects of $\mathrm{H}_{2}$ treatment acutely following surgical-induced brain injury. Since neurosurgical operations have been reported to cause postoperative cognitive dysfunction [21], it will be interesting to investigate the long term effects of $\mathrm{H}_{2}$ inhalation in the future, such as cognitive and memory functions following SBI.

\section{Acknowledgements}

This study was partially supported by grants from NIH NS53407 and NS43338 to JHZ

\section{Author details}

'Department of Neurosurgery, Loma Linda University Medical Center, 11234 Anderson Street, Loma Linda, CA 92354, USA. ${ }^{2}$ Department of Physiology and Pharmacology, Loma Linda University Medical Center, 11234 Anderson Street, Loma Linda, CA 92354, USA. ${ }^{3}$ Department of Anesthesiology, Loma Linda University Medical Center, 11234 Anderson Street, Loma Linda, CA 92354, USA.

\section{Authors' contributions}

JE carried out all the animal surgery, sample collection and drafted the manuscript. WC carried out all the neurological scoring tests and lipid peroxidation assay. VJ participated in the design of the study. FH participated in the study design and coordination. AC participated in the design of the study and coordination. JT participated in the study design. JZ participated in the design of the study and manuscript drafting. All authors read and approved the final manuscript.

\section{Competing interests}

The authors declare that they have no competing interests.

Received: 21 January 2011 Accepted: 18 May 2011

Published: 18 May 2011 


\section{References}

1. Andrews RJ, Muto RP: Retraction brain ischaemia: cerebral blood flow, evoked potentials, hypotension and hyperventilation in a new animal model. Neurol Res 1992, 14:12-8.

2. Hernesniemi JA, Leivo S: Management outcome in third ventricular colloid cysts in a defined population: A series of 40 patients treated mainly by transcallosal microsurgery. Surg Neurol 1996, 45:2-14.

3. Schroeder HW, Niendorf WR, Gaab MR: Complications of endoscopic third ventriculostomy. J Neurosurg 2002, 96:1032-40.

4. Hellwig D, Bertalanffy $H$, Bauer BL, Tirakotai W: Pontine hemorrhage. J Neurosurg 2003, 99:796-7.

5. Vachhrajani S, Fawaz C, Mathieu D, Menard C, Cusimano MD, Gentili F, Hodaie M, Kenny B, Kulkarni AV, Laperriere N, Schwartz M, Tsao M, Bernstein M: complications of Gamma Knife surgery: an early report from 2 canadian centers. J Neurosurg 2008, 109:2-7.

6. Menon D: critical care medicine: Management of raised intracranial pressure. In Oxford Textbook of Medicine. Volume 2.. 4 edition. Edited by: Warmell DA, Cox TM, Firth JD. New York: Oxford Univ Press; 2000.

7. Ohsawa I, Ishikawa M, Takahashi K, Watanabe M, Yamagata K, Katsura K, Katayama Y, Asoh S, Ohta S: Hydrogen acts as a therapeutic antioxidant by selectively reducing cytotoxic oxygen radicals. Nature Med 2007, 13:688-94.

8. Jadhav V, Solarglu I, Obenhaus A, Zhang JH: Neuroprotection against surgically induced brain injury. Surg Neurol 2007, 67:15-20.

9. Goldblum SE, Wu KM, Jay M: Lung myeloperoxidase as a measure of pulmonary leukostasis in rabbits. J Appl Physiol 1985, 59:1471-80.

10. Ohkawa $\mathrm{H}$, Ohishi $\mathrm{N}$, Yagi K: Assay for lipid peroxides in animal tissues by thiobarbituric acid reaction. Analytical Biochemistry 1979, 95:351-358.

11. Garcia JH, Wagner S, Liv KF, Hu XJ: Neurological deficit and extent of neuronal necrosis attributable to middle cerebral artery occlusion in rats. Statistical validation. Stroke 1995, 26:627-34.

12. Deletis V, Sala F: The role of intraoperative neurophysiology in the protection or documentation of surgically induced injury to the spinal cord. Ann N Y Acad Sci 2001, 939:137-144.

13. Lee ST, Chu K, Jung KH, Kim SJ, Kim DH, Kang KM, Hong NH, Kim JH, Ban JJ, Park HK, Kim SU, Park CG, Lee SK, Kim M, Roh JK: Anti-inflammatory mechanism of intravascular neural stem cell transplantation in haemorrhagic stroke. Brain 2008, 131:616-629.

14. Venetsanos AG, Huld T, Adams P, Bartzis JG: Source, dispersion and combustion modeling of an accidental release of hydrogen in an urban environment. J Haz Mat 2003, 105:1-25.

15. Simis A, Pires de Aguiar PH, Leite CC, Santana PA Jr, Rosemberg S, Teixeira MJ: Peritumoral brain edema in benign meningiomas: correlation with clinical, radiologic, and surgical factors and possible role on recurrence. Surg Neurol 2008, 70:471-7.

16. Matchett GA, Fathali N, Hasegawa Y, Jadhav V, Ostrowski RP, Martin RD, Dorotta IR, Sun $X$, Zhang $J H$ : Hydrogen gas is ineffective in moderate and severe neonatal hypoxia-ischemia rat models. Brain Res 2009, 1259:90-7.

17. Yamaguchi M, Jadhav V, Obenhaus A, Colohan A, Zhang JH: Matrix Metalloproteinase inhibition attenuates brain edema in an in vivo model of surgically-induced brain injury. Neurosurgery 2007, 61:1067-1076.

18. Waserman JK, Yang H, Schlichter LC: Glial responses, neuron death and lesion resolution after intracerebral hemorrhage in young vs. aged rats. Eur J Neurosci 2008, 28:1316-28.

19. Fontanari P, Badier M, Guillot C, Tomei C, Burnet H, Gardette B, Jammes Y: Changes in maximal performance of inspiratory and skeletal muscles during and after the $7.1 \mathrm{MPa} 10$ record human dive. Eur J Appl Physiol 2000, 81:325-328.

20. Wang ZF, Wang J, Zhang HY, Tang XC: Huperzine A exhibits antiinflammatory and neuroprotective effects in a rat model of transient focal cerebral ischemia. J Neurochem 2008, 106:1594-603.

21. Cao XZ, Ma H, Wang JK, Liu F, Wu BY, Tian AY, Wang LL, Tan WF: Postoperative cognitive deficits and neuroinflammation in the hippocampus triggered by surgical trauma are exacerbated in aged rats. Prog Neuropsychopharmacol Biol Psychiatry 2010, 34(8):1426-32.

doi:10.1186/2045-9912-1-7

Cite this article as: Eckermann et al: Hydrogen is neuroprotective against surgically induced brain injury. Medical Gas Research 2011 1:7.

\section{Submit your next manuscript to BioMed Central and take full advantage of:}

- Convenient online submission

- Thorough peer review

- No space constraints or color figure charges

- Immediate publication on acceptance

- Inclusion in PubMed, CAS, Scopus and Google Scholar

- Research which is freely available for redistribution

Submit your manuscript at www.biomedcentral.com/submit 